\title{
BIODEGRADAÇÃO DO HERBICIDA PROPANIL POR FUNGO ISOLADO DA RIZOSFERA DE ARROZ
}

\author{
CAMILA ORTIZ MARTINEZ* \\ CÉLIA MARIA MAGANHOTTO SOUZA E SILVA** \\ ALINE DE HOLANDA NUNES MAIA***
}

\begin{abstract}
Linhagens fúngicas isoladas da rizosfera de arroz foram avaliadas quanto ao seu potencial ligninolítico e algumas demonstraram aplicabilidade em estudos de degradação de agrotóxicos. O presente trabalho avaliou uma dessas linhagens quanto à capacidade de degradação do herbicida propanil e de seu metabólito 3,4 dicloroanilina (3,4-DCA). A linhagem P3SA1F foi cultivada em meio Czapek sólido, suplementado com duas concentrações de sacarose e diferentes concentrações de propanil e/ou 3,4-DCA $\left(2,5 ; 5,0 ; 10 ; 15 ; 20 ; 25\right.$ e $\left.50 \mu \mathrm{g} \mathrm{mL}^{-1}\right)$. Colônias que cresceram nesses meios foram avaliadas quanto à capacidade de degradar ambos os compostos em meio Czapeck líquido, suplementado nas concentrações de 2,5 e $25 \mu \mathrm{g} \mathrm{mL}^{-1}$. A determinação quantitativa dos resíduos dos herbicidas foi realizada por cromatografia a gás quantificando-se, paralelamente a biomassa formada. $O$ crescimento do fungo foi afetado proporcionalmente pelas doses aplicadas do herbicida e do metabólito. Observouse que a linhagem fúngica P3SA1F apresentou período de adaptação antes de iniciar a degradação do herbicida. Aos 28 dias de incubação obteve-se taxa de degradação de $66,4 \%$ para a concentração de $2,5 \mu \mathrm{g} \mathrm{mL}^{-1}$ e de $62,3 \%$ para a de $25 \mu \mathrm{g} \mathrm{mL}^{-1}$. Não foi detectada a presença de 3,4-DCA.
\end{abstract}

PALAVRAS-CHAVE: HERBICIDA - DEGRADAÇÃO; LINHAGEM FÚNGICA; PROPANIL; 3,4-DCA.

* Engenheira Agrônoma, Mestranda da Faculdade de Engenharia de Alimentos, Universidade de Campinas (UNICAMP), Campinas, SP (e-mail: camila@fea.unicamp.br).

** Bióloga, Doutora em Microbiologia Ambiental, Pesquisadora da Embrapa Meio Ambiente, Jaguariúna, SP, Brasil (e-mail: celia@cnpma.embrapa.br).

*** Engenheira Agrônoma, Doutora em Estatística, pesquisadora da Embrapa Meio Ambiente (e-mail: ahmaia@cnpma.embrapa.br). 


\section{INTRODUÇÃO}

O propanil (3',4'-dicloropropionanilina), herbicida seletivo de contato e pós-emergente (derivado das cloroanilidas), tem sido utilizado intensivamente na cultura do arroz (Oryza sativa L.) para o controle de plantas daninhas de folha larga e estreita (ALAWI, 1982). Com meia-vida de aproximadamente 1 ano, sofre clivagem hidrolítica em meio fortemente ácido e alcalino originando 3,4dicloroanilina (3,4-DCA) (Figura 1) e ácido propiônico. O propanil é estável entre pH 4 e 9 (TOMLIN, 2000).

\section{FIGURA 1 - ESTRUTURA DO HERBICIDA PROPANIL (A) E SEU METABÓLITO 3,4-DCA (B)}

A<smiles>CCC(=O)Nc1ccc(Cl)c(Cl)c1</smiles>

B<smiles>Nc1ccc(Cl)c(Cl)c1</smiles>

As cloroanilinas adsorvem-se às substâncias húmicas presentes no solo, podendo permanecer ligadas durante anos após a aplicação. Também estão sujeitas à dimerização e polimerização formando compostos azo, altamente estáveis e resistentes à mineralização (BRUNSBACH e REINEKE, 1993; KREMER e STERNER, 1996).

Várias bactérias, algumas isoladas de cultivo de arroz, foram selecionadas pela capacidade de degradar cloroanilinas, tais como Comamonas testosteroni (BOON et al., 2000), Pseudomonas acidovorans BN3.1 (BRUNSBACH e REINEKE, 1993), Proteus mirabilis (CORREA e STEEN, 1995), Aquaspirillum sp e Paracoccus denitrificans 3CA (SUROVTSEVA et al., 1996). A bactéria Pseudomonas fluorescens biotipo II hidrolisou propanil à 3,4-DCA (ZABLOTOWICZ et al., 2001).

Entre os fungos, a espécie Phanerochaete chrysosporium foi responsável pela degradação de 3,4-DCA em meio de cultura com limitação de nitrogênio (N). No processo, os principais metabólitos formados foram $N$-(3,4-diclorofenil)- $\alpha$-cetoglutaril- $\delta$-amida (DCAX) e DCA-succinimida (DCASI), o qual apresentou concentração máxima aos 10 dias de incubação. O DCAX também foi detectado em meios de cultura com limitação de carbono (C), ou suplementação de N e C (SANDERMANN et al., 1998), mas os autores não encontraram correlação entre a atividade da enzima lignina peroxidase e a degradação de 3,4-DCA. Outros fungos têm sido envolvidos na degradação de 3,4-DCA como o basidiomiceto Filoboletus TA9054, cujo metabólito formado em maior quantidade no processo foi 0 3,3',4,4'-tetracloroazobenzeno (TCAB) (KREMER e STERNER, 1996).

SILVA, MELO e VIEIRA (2003) isolaram linhagens fúngicas, consideradas degradadoras em potencial de compostos orgânicos em culturas de arroz irrigado do estado de Santa Catarina. Verificaram o potencial ligninolítico dessas linhagens e constataram que apenas duas (P3SA1F e P11SA2F) poderiam ter aplicabilidade em estudos de degradação de compostos orgânicos. O presente trabalho teve como objetivo avaliar o potencial de uma dessas linhagens pré-selecionadas para a degradação do herbicida propanil e de seu metabólito 3,4-DCA.

\section{MATERIAL E MÉTODOS}

\subsection{CRESCIMENTO MICELIANO (CM)}

Cultivou-se a linhagem P3SA1F em meio Czapek sólido (3 g L-1 $\mathrm{NaNO}_{3}, 1 \mathrm{~g} \mathrm{~L}^{-1} \mathrm{~K}_{2} \mathrm{HPO}_{4}$, $0,5 \mathrm{~g} \mathrm{~L}^{-1} \mathrm{MgSO}_{4} \cdot 7 \mathrm{H}_{2} \mathrm{O}, 0,5 \mathrm{~g} \mathrm{~L}^{-1} \mathrm{KCl}, 0,01 \mathrm{~g} \mathrm{~L}^{-1} \mathrm{FeSO}_{4} \cdot 7 \mathrm{H}_{2} \mathrm{O}, 16 \mathrm{~g} \mathrm{~L}^{-1}$ ágar), suplementado com sacarose 
(0 e $10 \mu \mathrm{g} \mathrm{mL}^{-1}$ ) e diferentes concentrações de propanil ou 3,4-DCA $(2,5 ; 5,0 ; 10 ; 15 ; 20 ; 25$ e $50 \mu \mathrm{g} \mathrm{mL}^{-1}$ ). Avaliou-se o desenvolvimento das colônias após 2, 4, 6, 7, 9, 11 e 14 dias da semeadura, registrando nessas datas o diâmetro da colônia em cada placa (medido com régua). Taxas médias do crescimento miceliano foram calculadas para cada unidade experimental, dividindo-se a diferença entre o acréscimo no diâmetro miceliano entre os dias 2 e 14 pelo intervalo de tempo (12 dias) (SILVA, 1996).

Utilizou-se no experimento, o delineamento estatístico completamente casualisado com cinco repetições. Os efeitos das doses de sacarose, do herbicida, ou do 3,4-DCA, e suas influências sobre a taxa de crescimento miceliano (TCM) foram investigados mediante análise de variância e testes $\mathrm{F}$ de Snedecor (MONTGOMERY, 1991). Em cada ensaio foram ajustados modelos de regressão (DRAPER e SMITH, 1981) para descrever o efeito da dose do composto orgânico avaliado sobre a TCM.

\subsection{BIODEGRADAÇÃO DO HERBICIDA}

Três discos de crescimento $(\varnothing=5 \mathrm{~mm})$ da linhagem fúngica P3SA1F foram inoculados em meio de cultura Czapek líquido, suplementado com $10 \mathrm{~g} \mathrm{~L}^{-1}$ de sacarose e propanil nas concentrações de campo $\left(2,5 \mu \mathrm{g} \mathrm{mL}^{-1}\right)$ e dez vezes superior a essa $\left(25 \mathrm{\mu g} \mathrm{mL}^{-1}\right)$. As culturas foram incubadas em shaker (New Brunswick Cientific, modelo inova) a 28으 $\mathrm{C}$ sob agitação constante (160 rpm). O tratamento controle constou de meio de cultura sem microrganismo nas mesmas condições (SILVA, 1996). Utilizouse delineamento experimental inteiramente casualizado, sendo os fatores fungo (presença e ausência) e tempos de avaliação (2, 4, 8, 18 e 28 dias após incubação) arranjados em esquema fatorial.

Extraiu-se o propanil do meio de cultura mediante extração líquido-líquido. Adicionaram-se ao meio $30 \mathrm{~mL}$ de diclorometano:acetona (1:1), ambos grau p.r.), recolhendo-se a fase orgânica por duas vezes. A fase orgânica foi evaporada em rotaevaporador $(\mathrm{BUCHI})$ até a secura, retomando-se o resíduo em $10 \mathrm{~mL}$ de isooctano (2,2,4 dimetilpentano) grau p.r. Realizou-se a determinação quantitativa do propanil em cromatógrafo a gás, modelo HP 5890, com detector de captura de elétrons. As condições cromatográficas foram: nitrogênio $\left(\mathrm{N}_{2}\right)$ como gás de arraste; fluxo de $5,4 \mathrm{~mL} \mathrm{~min}^{-1}$; temperatura do detector $300^{\circ} \mathrm{C}$; temperatura do injetor $270^{\circ} \mathrm{C}$; coluna HP-1 $(30 \mathrm{~m} \times 0,53 \mathrm{~mm} \times 1,5 \mu \mathrm{m})$; temperatura do forno de $80^{\circ} \mathrm{C}$ ( $1 \mathrm{~min}$ ) e $25^{\circ} \mathrm{C} \mathrm{min}^{-1}$ até $270^{\circ} \mathrm{C}$ (10 min).

Quantifiou-se 3,4-DCA aos 18 e 28 dias após incubação. O metabólito foi destilado-extraído de $20 \mathrm{~mL}$ de meio de cultura adicionado de $230 \mathrm{~mL}$ de água deionizada, utilizando-se condensador do tipo Bleidner, sendo recolhido em $100 \mathrm{~mL}$ de isooctano adicionado de $1,5 \mathrm{~mL}$ de ácido sulfúrico $1 \mathrm{~mol} \mathrm{~L}^{-1}$. Após 4 horas de destilação-extração realizou-se a extração líquido-líquido com isooctano. Recolheu-se a fase orgâncica e ajustou-se o pH da fase aquosa para 7,0 (utilizando-se solução de bicarbonato de sódio saturada). Essa foi extraída quatro vezes com $25 \mathrm{~mL}$ de isooctano, recolhendose a fase orgânica. Evaporou-se todo solvente recolhido até o volume de $2 \mathrm{~mL}$, trocando-se o solvente por duas vezes com adição de $5 \mathrm{~mL}$ de tolueno (grau p.r.) e ressuspendendo o volume final de $10 \mathrm{~mL}$ de tolueno.

A quantificação de 3,4-DCA ocorreu em cromatógrafo a gás, modelo HP 6890, com detector termoiônico específico para nitrogênio e fósforo (NPD). As condições cromatográficas foram: hélio como gás de arraste; fluxo de $6 \mathrm{~mL} \mathrm{~min}^{-1}$; temperatura do detector $325^{\circ} \mathrm{C}$; temperatura do injetor $260^{\circ} \mathrm{C}$; coluna DB-1 (30 m x 0,32 mm $\left.\times 1 \mu \mathrm{m}\right)$; temperatura do forno $60^{\circ} \mathrm{C} / 1$ minuto, $25^{\circ} \mathrm{C} \mathrm{min}^{-1}$ até $250^{\circ} \mathrm{C}$.

Os cálculos para quantificação do propanil e de 3,4-DCA foram baseados em curvas de calibração, obtidas para soluções padrões em concentrações conhecidas (Figura 2). Para cada análise cromatográfica realizada, referente aos períodos de incubação, construiu-se a curva de calibração utilizando a faixa de trabalho de 0,05 a $1,0 \mathrm{\mu g} \mathrm{mL}^{-1}$, cujos picos foram integrados pela altura. Para todas as curvas de calibração o coeficiente de correlação mostrou-se maior ou igual a 0,99. 


\section{FIGURA 2 - CURVAS DE CALIBRAÇÃO DO HERBICIDA PROPANIL (A) E DO SEU METABÓLITO 3,4-DICLOROANILINA (B)}

A

Curva de calibração de Propanil

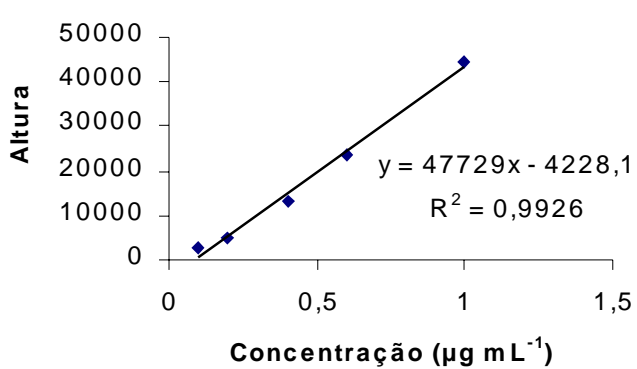

B

Curva de calibração da 3,4 -DCA

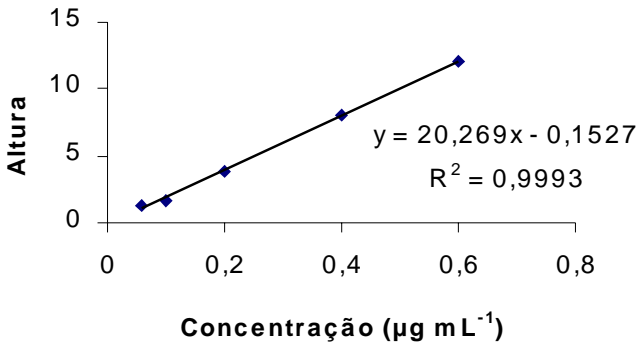

Amostras fortificadas $\left(2,5 \mu \mathrm{g} \mathrm{mL}^{-1}\right.$ de propanil e $25 \mu \mathrm{g} \mathrm{mL}^{-1}$ de $\left.3,4-\mathrm{DCA}\right)$ e amostras em branco foram submetidas aos mesmos procedimentos de extração para verificar a eficiência dos métodos e a existência de interferentes nas matrizes estudadas e nos materiais e reagentes utilizados.

A avaliação da biomassa fúngica ocorreu paralelamente ao experimento de degradação. $A$ biomassa foi filtrada em funil de Büchner e seca em estufa a $55^{\circ} \mathrm{C}$ até peso constante.

\section{RESULTADOS E DISCUSSÃO}

Avaliou-se o crescimento da linhagem fúngica P3SA1F, tanto na presença do herbicida propanil quanto de seu metabólito 3,4-DCA. Observou-se que ambas as moléculas inibiram o crescimento fúngico, proporcionalmente à dose aplicada. Na dose máxima $\left(50 \mu \mathrm{g} \mathrm{mL}^{-1}\right)$, tanto na presença como na ausência de sacarose, a linhagem apresentou inibição média na TCM de 80,25 e 91,9\% na presença de propanil e de 3,4-DCA, respectivamente. Verificou-se redução média na TCM em relação ao controle de 37,3 e 65,1\% na presença de 3,4-DCA (15, 20 e $25 \mu \mathrm{g} \mathrm{mL}^{-1}$ ) sem e com adição de sacarose, respectivamente. O mesmo foi observado para o propanil com redução de $29 \%$ na ausência de sacarose para a dose $25 \mu \mathrm{g} \mathrm{mL}^{-1}$ e, em média, de $47,8 \%$ na presença de sacarose para as doses $20 \mathrm{e}$ $25 \mu \mathrm{g} \mathrm{mL}{ }^{-1}$.

As estimativas dos parâmetros dos modelos de regressão ajustados para descrever a variação nas taxas de crescimento micelial em função das doses dos xenobióticos são apresentados nas Tabelas 1 e 2 .

\section{TABELA 1 - ESTIMATIVAS DOS PARÂMETROS DOS MODELOS DE REGRESSÃO LINEAR QUE DESCREVEM VARIAÇÕES NAS TCM DA LINHAGEM FÚNGICA P3SA1F EM FUNÇÃO DE DOSES DO PROPANIL EM MEIOS DE CULTURA COM DIFERENTES CONCENTRAÇÕES DE SACAROSE}

\begin{tabular}{clcccc}
\hline $\begin{array}{l}\text { Dose de } \\
\text { sacarose }\left(\mathbf{g L}^{-1}\right)\end{array}$ & $\begin{array}{l}\text { Variável } \\
\text { dependente }\end{array}$ & Parâmetro & $\begin{array}{l}\text { Estimativa } \\
(\mathbf{m m})\end{array}$ & $\begin{array}{l}\text { Erro-padrão } \\
(\mathbf{m m})\end{array}$ & Valor $\mathbf{p}^{*}$ \\
\hline \multirow{2}{*}{0} & $\begin{array}{l}\text { Taxa de } \\
\text { crescimento (Tx) }\end{array}$ & $\beta$ & 5.2880 & 0,1050 & $<0,0001$ \\
& & $\alpha$ & -0.0880 & 0,0045 & $<0,0001$ \\
10 & $\log (\mathrm{Tx})$ & $\beta$ & $-0,7534$ & 0,0107 & $<0,0001$ \\
& & $\alpha$ & $-0,0180$ & 0,0004 & $<0,0001$ \\
\hline
\end{tabular}

*Níveis de significância nominais associados aos testes $\mathrm{t}$ bilaterais para as hipóteses $\alpha=0$ ou $\beta=0$. 
TABELA 2 - ESTIMATIVAS DOS PARÂMETROS DOS MODELOS DE REGRESSÃO LINEAR QUE DESCREVEM VARIAÇÕES NAS TCM DA LINHAGEM FÚNGICA P3SA1F EM FUNÇÃO DE DOSES DE 3,4-DCA EM MEIOS DE CULTURA COM DIFERENTES CONCENTRAÇÕES DE SACAROSE

\begin{tabular}{cccccc}
\hline $\begin{array}{c}\text { Dose de } \\
\text { sacarose }\left(\mathbf{g L}^{-1}\right)\end{array}$ & $\begin{array}{c}\text { Variável } \\
\text { dependente }\end{array}$ & Parâmetro & $\begin{array}{c}\text { Estimativa } \\
(\mathbf{m m})\end{array}$ & $\begin{array}{c}\text { Erro-padrão } \\
(\mathbf{m m})\end{array}$ & $\begin{array}{c}\text { Valor } \\
\mathbf{p}^{*}\end{array}$ \\
\hline 0 & $\begin{array}{c}\text { Taxa de } \\
\text { crescimento } \\
(\mathrm{Tx})\end{array}$ & $\alpha$ & 4,6471 & 0,0993 & $<0,0001$ \\
& $\mathrm{~L}(\mathrm{Tx})$ & $\alpha$ & $-0,0765$ & 0,0665 & $<0,0001$ \\
10 & $\mathrm{Log}(\mathrm{T})$ & 0,7069 & 0,0206 & $<0,0001$ \\
\hline
\end{tabular}

*Níveis de significância nominais associados aos testes t bilaterais para as hipóteses $\alpha=0$ ou $\beta=0$.

Os efeitos das doses de sacarose, propanil ou 3,4-DCA e suas interações foram significativos (Teste $F, p<0,0001$ ). Os coeficientes de variação mostraram-se inferiores a $8 \%$, indicando que grande parte da variabilidade da taxa de crescimento miceliano foi explicada pelos fatores avaliados.

Observou-se que a taxa diária de crescimento na ausência de sacarose foi maior do que na sua presença, sendo que o padrão de variação da TCM em função da dose dos xenobióticos revelou-se linear para a dose zero e exponencial negativo para a dose de $10 \mu \mathrm{g} \mathrm{mL}^{-1}$ de sacarose (Figura 3).

FIGURA 3 - TAXAS MÉDIAS DIÁRIAS DE CRESCIMENTO MICELIANO DO FUNGO P3SA1F EM FUNÇÃO DE DOSES DE PROPANIL (A) E DE 3,4-DCA (B) EM MEIOS DE CULTURA SEM E COM ADIÇÃO DE SACAROSE, CONSTANDO O CONTROLE SOMENTE DE SACAROSE $30 \mathrm{~g} \mathrm{~L}^{-1}$ COMO FONTE DE CARBONO

A

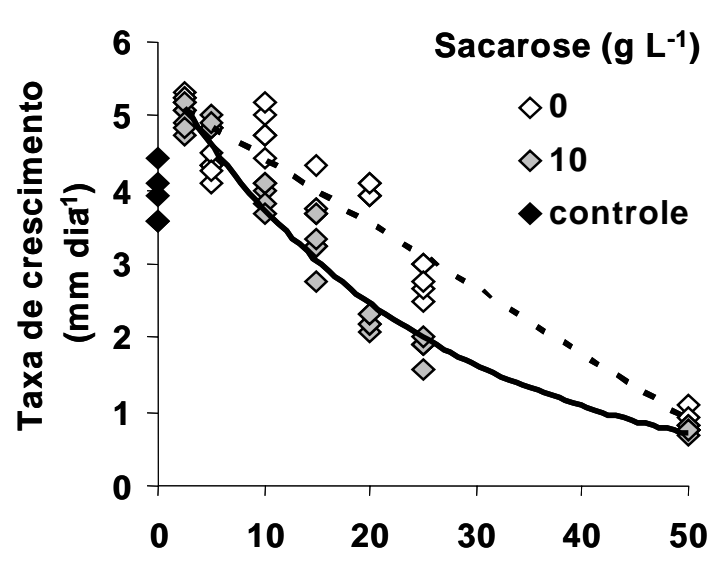

Concentração de Propanil $\left(\mu g^{-1}\right)$
B

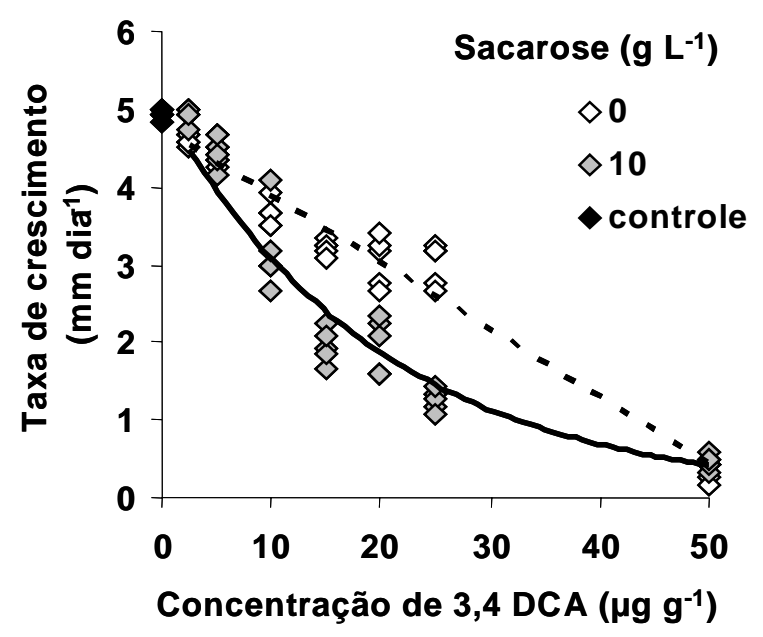

--------Tendência linear — Tendência exponencial 
Nas condições cromatográficas estabelecidas, o propanil foi identificado como o pico com tempo de retenção médio de 7,45 min e 3,4-DCA como o pico com tempo de retenção médio de 6,53 min (Figura 4). Não houve interferência das matrizes estudadas e dos materiais e reagentes utilizados na análise de ambos os compostos. Para o propanil obteve-se recuperação de $87 \%$ da amostra fortificada a $2,5 \mu \mathrm{g} \mathrm{mL} \mathrm{m}^{-1}$, enquanto para 03,4 -DCA a recuperação foi de $90 \%$ da amostra fortificada a $25 \mu \mathrm{g} \mathrm{mL}$.

\section{FIGURA 4 - CROMATOGRAMA TÍPICO OBTIDO PARA PROPANIL (A) E 3,4-DICLOROANILINA (B) VIA CROMATOGRAFIA A GÁS}

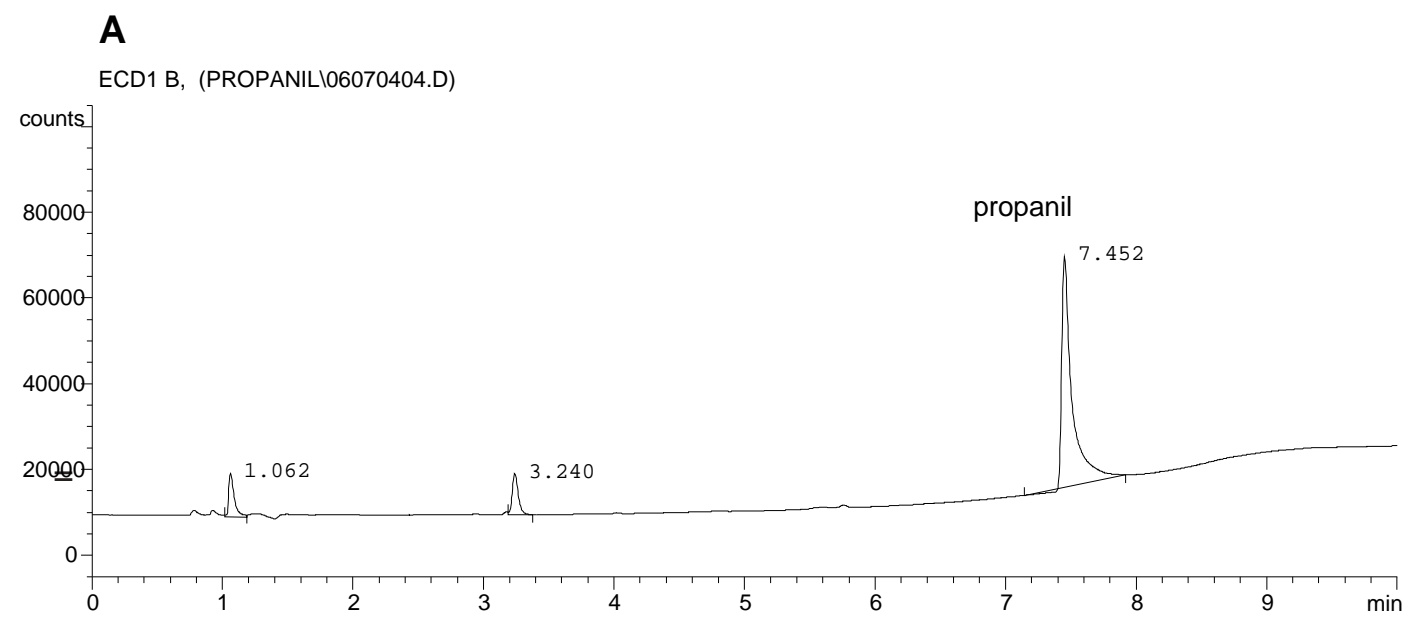

B

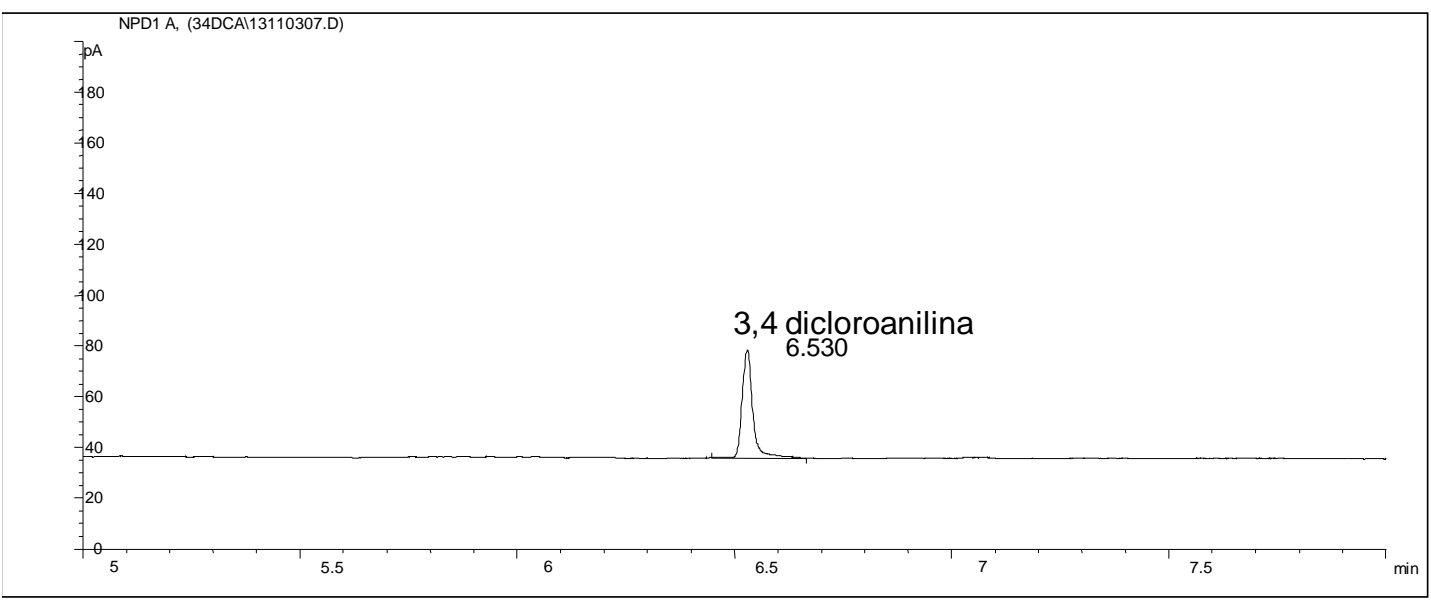

$\mathrm{Na}$ avaliação da degradação do herbicida, a linhagem fúngica P3SA1F apresentou período de adaptação de aproximadamente 8 dias, o qual está representado graficamente na Figura 5 pela fase "lag". Posteriormente, na fase de enriquecimento, observou-se taxa de degradação mais alta na maior concentração do herbicida ( $\left.25 \mu \mathrm{g} \mathrm{mL}^{-1}\right)$, chegando a 62,3\% aos 18 dias de incubação, enquanto na menor dose $\left(2,5 \mu \mathrm{g} \mathrm{mL}^{-1}\right)$ a taxa foi de apenas $38,9 \%$ no mesmo período. No entanto, aos 28 dias ambas as doses apresentaram taxas de degradação semelhantes, ou seja, 66,4 e 67,2\% para a menor e maior dose, respectivamente (Figura 5). As estimativas de meia-vida para as doses 2,5 e $25 \mathrm{\mu g} \mathrm{mL}^{-1}$ foram 20,83 e 14,42 dias, respectivamente (Figura 6). 

$\left(\mu \mathrm{gL}^{-1}\right)$, MEDIDA DURANTE O EXPERIMETNTO DE DEGRADAÇÃO EM CONJUNTO COM A RECUPERAÇÃO DO PROPANIL (\%) AO LONGO DO TEMPO

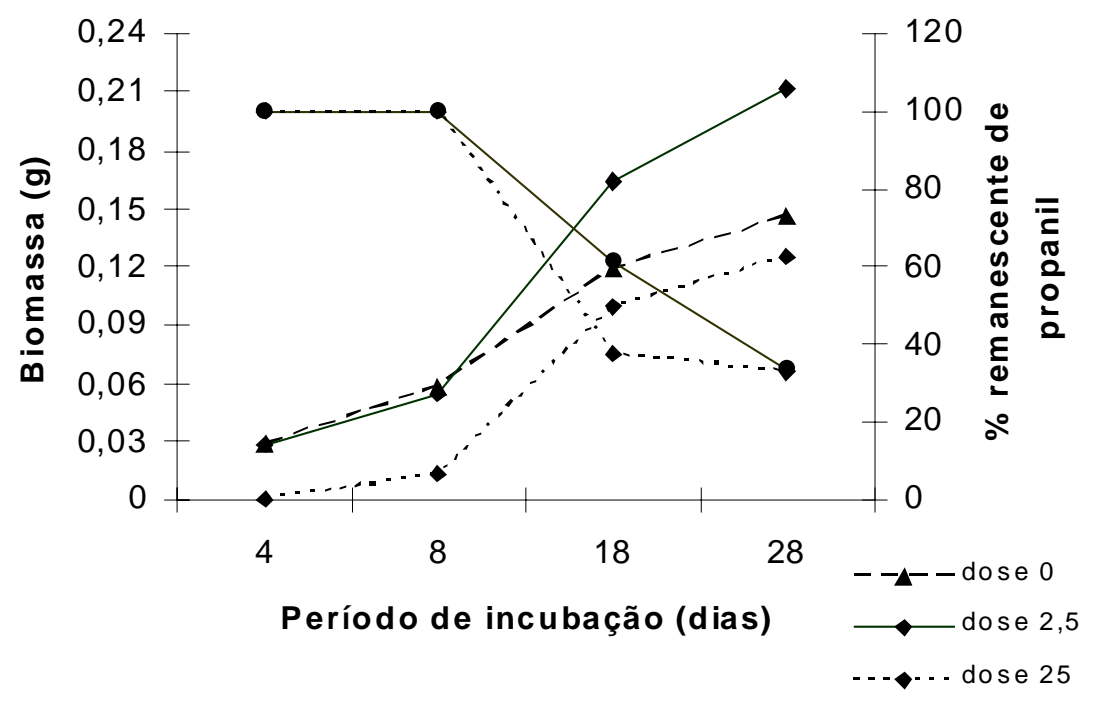

No experimento de degradação verificou-se diferença significativa entre o efeito das doses sobre a taxa de crescimento da biomassa (Teste $F, p<0,0001$ ), assim como entre as esmimativas de contraste entre as taxas médias de crescimento da biomassa. Na dose de 2,5 $\mathrm{g} \mathrm{mL}^{-1}$ obteve-se taxa 2,357 maior do que na dose zero e 3,107 maior do que a taxa na dose de $25 \mu \mathrm{gL}^{-1}$ (Tabela 3). Os coeficientes de variação foram inferiores a 2,4\%.

FIGURA 6 - CURVAS DE DEGRADAÇÃO DO PROPANIL (DOSE 2,5 E $25 \mu \mathrm{g} \mathrm{mL}^{-1}$ ) EM MEIO DE CULTURA INDICANDO A MEIA-VIDA DO PRODUTO PARA AMBAS AS DOSES

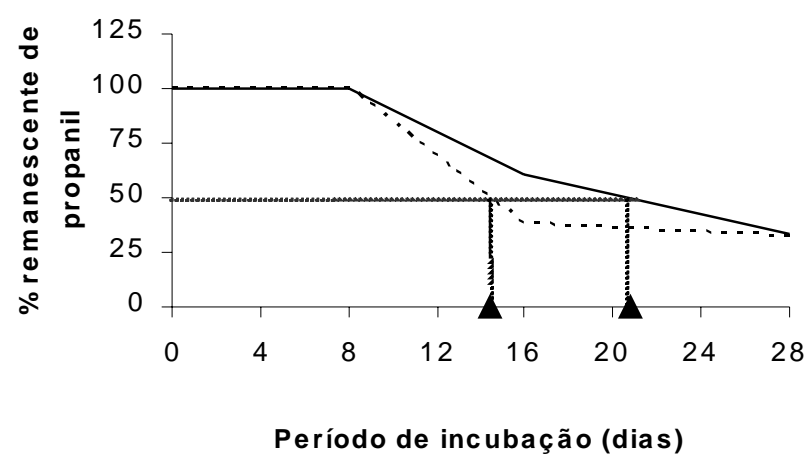

$-2,5 \mu \mathrm{g} \mathrm{mL}^{-1}------25 \mu \mathrm{gL}^{-1} \quad \Delta$ Meia-vida 


\title{
TABELA 3 - ESTIMATIVAS DE CONTRASTES ENTRE TAXAS MÉdIAS DE CRESCIMENTO DA BIOMASSA, RESPECTIVOS ERROS-PADRÃO E RESULTADOS DOS TESTE F
}

\begin{tabular}{cccc}
\hline Contraste & Estimativa & Erro-padrão & Pr $>\mathbf{F}$ \\
\hline $2,5 \mu \mathrm{gL}^{-1} \times$ dose zero & 2,357 & 0,11 & $<0,0001$ \\
$25 \mu \mathrm{mL}^{-1} \times$ dose zero & $-0,750$ & 0,11 & 0,0006 \\
$25 \mu \mathrm{gL}^{-1} \times 2,5 \mu \mathrm{gL}^{-1}$ & $-3,107$ & 0,11 & $<0,0001$ \\
\hline
\end{tabular}

Comparando os resultados obtidos com os de KREMER e STERNER (1996) verifica-se que a linhagem fúngica PSAP31F degradou parcialmente o produto $( \pm 67 \%)$ em 28 dias. Não foi detectada a presença do metabólito 3,4-DCA, provavelmente devido a rápida mineralização do mesmo. No entanto, o fungo Basidiomycete filoboletus TA9054 reduziu após 15 dias a concentração inicial de 3,4-DCA de 1,2 mM para 0,2 mM (83\%). Tal concentração inibiu o crescimento fúngico, mas não afetou 0 metabolismo do composto (KREMER e STERNER, 1996). Já o fungo Phanerochaete chrysosporium degradou 3,4-DCA à N-(3,4-diclorofenil)- $\alpha$-cetoglutaril- $\delta$-amida. Esse metabólito esteve presente até 21 dias de incubação (SANDERMANN et al., 1998).

\section{CONCLUSÃO}

O herbicida propanil quando aplicado na dose de campo $\left(2,5 \mathrm{\mu g} \mathrm{mL}^{-1}\right)$ estimulou o crescimento fúngico. Já o aumento da dose a partir de $15 \mu \mathrm{g} \mathrm{mL}^{-1}$, tanto para a molécula parental, quanto para o metabólito (3,4-DCA) inibiu o mesmo. Foi observada degradação de ambas as moléculas na presença da linhagem P3SA1F, demonstrado pela redução de $56 \%$ na concentração de propanil em 18 dias. A meia-vida para o composto parental foi de 20,83 e 14,42 dias para as concentrações 2,5 e $25 \mathrm{\mu g} \mathrm{mL}^{-1}$, respectivamente.

\begin{abstract}
BIODEGRADATION OF THE HERBICIDE PROPANIL BY FUNGI ISOLATED FROM RICE RHIZOSPHERE Fungal strains isolated from rice rhizosphere were evaluated towards its lignolytic potential, and some of them showed applicability in studies of pesticide degradation. The present study evaluated one of those strains for its ability to degrade propanil and its metabolite 3,4-dichloroaniline (3,4-DCA). The P3SA1F strain was grown in solid Czapek medium supplemented with two sucrose concentrations and different propanil and/or 3,4-DCA concentrations $\left(2.5 ; 5.0 ; 10.0 ; 15.0 ; 25.0\right.$ and $\left.50.0 \mu \mathrm{g} \mathrm{mL}^{-1}\right)$. Colonies that grew in these medium were evaluated for their ability to degrade both compounds in liquid Czapek medium, supplemented with propanil at concentrations of 2.5 and $25.0 \mathrm{\mu g} \mathrm{ml}^{-1}$. The quantitative determination of the herbicides residues was realized by gas chromatography, simultaneously the biomass formed was quantified. The fungi growth was proportionally affected by the applied doses of the herbicide and metabolite. It was observed that the fungal P3SA1F strain showed an adaptation phase before the beginning of herbicide degradation. In 28 days of incubation the degradation rates of $66.41 \%$ and $62.34 \%$ were observed for propanil concentrations of 2.5 and $25 \mathrm{\mu g} \mathrm{ml}^{-1}$ respectively. 3,4-DCA was not observed in the propanil degradation studies.
\end{abstract}

KEY-WORDS: HERBICIDE - DEGRADATION; FUNGAL STRAIN; PROPANIL; 3,4 DCA.

\section{REFERÊNCIAS}

1 ALAWI, M. A. Determination of propanil in rice, potatoes and water by HPLC. Fresenius Z. Analitical Chemistry, v.312, p.536-538, 1982. 
2 BOON, N.; GORIS, J.; PAUL, V.; VERSTRAETE, W.; TOP, E. M. Bioaugmentation of activated sludge by an indigenous 3-chloroaniline-degrading Comamonas testosterone strain, $12 \mathrm{gfp}$. Applied and Environmental Microbiology, v. 66, n. 7, p. 2906-2913, 2000.

3 BRUNSBACH, F. R.; REINEKE, W. Degradation of chloroanilines in soil slurry by specialized organisms. Applied Microbiology and Biotechnology, v. 40, p. 402-407, 1993.

4 CORREA, I. E.; STEEN, W. C. Degradation of propanil by bacterial isolates and mixed populations from a pristine lake. Chemosphere, v.30, n.1, p.103-116, 1995.

5 DRAPER, N.; SMITH, H. Applied regression analysis. $2^{\text {nd }}$ ed. New York: John Wiley \& Sons, 1981. $709 \mathrm{p}$.

6 KREMER, S.; STERNER, O. Metabolism of 3,4-Dichloroaniline by the Basidiomycete Filoboletus Species TA9054. Journal Agricultural Food Chemistry, v.44, p.1155-1159, 1996.

7 MONTGOMERY, D. C. Design and analysis of experiments. $3^{\text {rd }}$ ed. New York : John Wiley \& Sons, 1991. $672 \mathrm{p}$.

8 SANDERMANN JR, H.; HELLER, W.; HERTKORN, N.; HOQUE, E. PIEPER, D.; WINKLER, R. A new intermediate in the mineralization of 3,4-Dichloroaniline by the white rot fungus Phanerochaete chrysosporium. Applied and Environmental Microbiology, v. 64, n. 9, p. 3305-3312, 1998.

9 SILVA, C. M. M. S. Biodegradação do fungicida carbendazim. Rio Claro, 1996. 86 p. Dissertação (Mestrado), Universidade Estadual Paulista.

10 SILVA, C. M. M. S.; MELO, I. S.; VIEIRA, R. F. Production of phenol-oxidases and peroxidases by fungi isolated from irrigated rice. Brazilian Journal of Microbiology, n.34, p.53-55, 2003.

11 SUROVTSEVA, E. G.; IVOILOV, V. S.; VASILEVA, G. K.; BELYAEV, S. S. Degradation of chlorinated anilines by certain representatives of the genera Aquaspirilum and Paracoccus. Microbiology, v. 65, p.553-559, 1996.

12 TOMLIN, C. D. S. (Ed.). The pesticide manual: a world compendium. $12^{\text {th }}$ ed. Surrey: British Crop Protection Council, 2000. 1250 p.

13 ZABLOTOWICZ, R. M.; LOCKE, M. A.; HOAGLAND, R. E.; KNIGHT, S. S.; CASH, B. Fluorescent Pseudomonas isolates from Mississipi Delta oxbow lakes: in vitro herbicide biotransformations. Environmental Toxicology, v.16, n.1, p.9-19, 2001.

14 ZAKHAROVA, E. A.; SHCHERBAKOV, A. A.; BRUDNIK, V. V.; SKRIPKO, N. G.; BULKHIN, N. S.; IGNATOV, V. V. Biosynthesis of indole-3-acetic acid in Azospirillum brasilense. Eur. Journal Biochemistry, n.259, p.572-576, 1999. 